\title{
Clinical evaluation of double-channel gastroscope for endoscopic retrograde cholangiopancreatography in patients with Billroth II gastrectomy
}

\author{
Sheng Wang, Wen Liu, Siyu Sun, Guoxin Wang, Xiang Liu, Nan Ge, Jintao Guo
}

Shengjing Hospital affiliated to China Medical University, Shenyang, China

Gastroenterology Rev 2016; 11 (3): 163-169

DOI: $10.5114 /$ pg.2016.61370

Key words: Billroth II gastrectomy, double-channel gastroscope, endoscopic retrograde cholangiopancreatography.

Address for correspondence: Prof. Siyu Sun MD, PhD, Shengjing Hospital affiliated to China Medical University, Endoscopy Center, 110004 Shenyang, China, phone: +86 18940255115, e-mail: sunsiyu-mail@sohu.com

\begin{abstract} retrograde cholangiopancreatography (ERCP) and interventions. working channel (4.2-mm diameter, 2.8-mm diameter). (83.3\%), and no serious complications were encountered. tography in patients with Billroth II gastrectomy.

\section{Introduction}

Biliary diseases are common in patients with Billroth II gastrectomy, although there are some difficulties in performing endoscopic retrograde cholangiopancreatography (ERCP) and associated procedures because of the altered anatomy. Difficulties are encountered especially in entering the afferent loop, accessing the papilla, retrograde cannulation, and performing endoscopic sphincterotomy (EST), resulting in a lower success rate and a higher incidence of complications compared to patients with normal anatomy. We have carried out ERCP with a double-channel gastroscope in patients with Billroth II gastrectomy, and good efficacy was obtained.
\end{abstract}

Aim: To evaluate the use of a double-channel gastroscope in patients with Billroth II gastrectomy to perform endoscopic

Material and methods: From January 2008 to December 2013, 18 patients with Billroth II gastrectomy were enrolled in this study. Endoscopic retrograde cholangiopancreatography was performed using a straight forward gastroscope with double

Results: The success rate of selective cannulation and accomplishment of planned procedures was 15 out of 18 patients

Conclusions: The double-channel gastroscope appears to be useful in performing endoscopic retrograde cholangiopancrea-

\section{Aim}

In this paper, we present our strategy and experiences of 18 cases of ERCP in patients with Billroth II gastrectomy.

\section{Material and methods \\ Endoscope and accessories}

Endoscope: PENTAX EG-3831T, Sphincterotome, Nasal Biliary Drainage Catheter and Guidewire: Cook MEDICAL, Needle Knife and Basket: Olympus, Extraction Balloon: ENDO-FLEX, Metal stent and Dilation balloon: Boston Scientific Corporation.

\section{Patients}

Between January 2008 and December 2013, 18 consecutive patients with Billroth II gastrectomy (15 males, 3 females) were admitted to the Endoscopy Centre of the China Medical University because of biliary diseases. The mean age of the 18 patients was $67 \pm 11.7$ (42-82) years and the male to female ratio was $5: 1$. These patients' characteristics are shown in Table I. The surgical records of all patients were reviewed or they were given upper gastrointestinal contrast for confirmation of surgical operations for the stomach. 
Table I. Patients' characteristics

\begin{tabular}{lc}
\hline Characteristic & Value \\
\hline Age [years]: & $67 \pm 11.7$ \\
\hline Mean & $42-82$ \\
\hline Range & 15 \\
\hline Gender: & 3 \\
\hline Male & 10 \\
\hline Female & 8 \\
\hline Reasons of Billroth II gastrectomy: & 16 \\
\hline Gastric cancer & 2 \\
\hline Peptic ulcer & 1 \\
\hline Types of Billroth II gastrectomy: & 6 \\
\hline Antecolic gastrojejunostomy & 9 \\
\hline Retrocolic gastrojejunostomy & 2 \\
\hline Diagnosis: & \\
\hline Choledocholithiasis & \\
\hline Gallstones + choledocholithiasis & \\
\hline Bile duct cancer & \\
\hline Carcinoma of papilla of Vater & \\
\hline
\end{tabular}

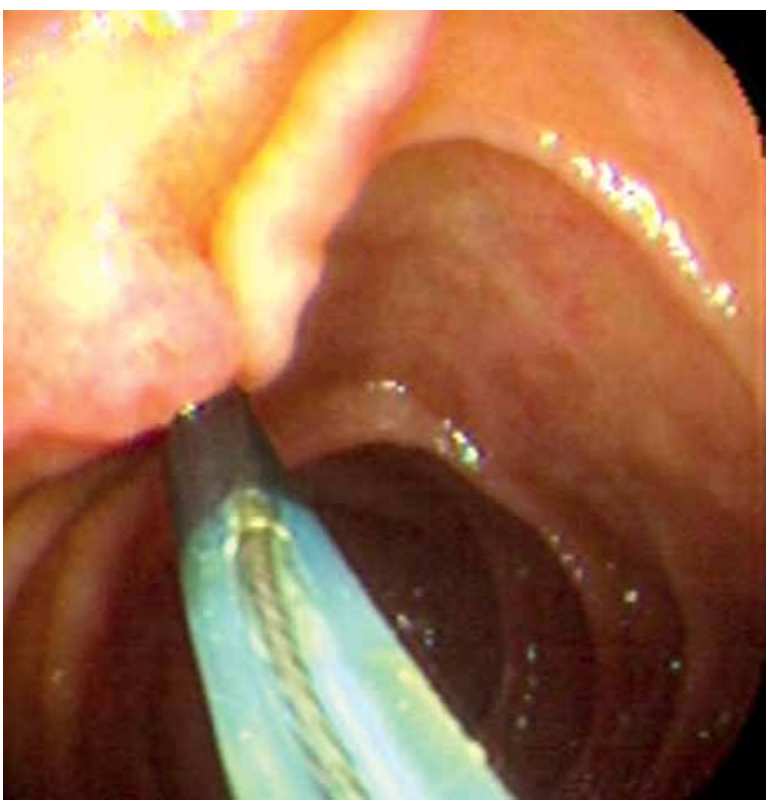

Figure 1. Gastroscope could be delivered to the descending segment of duodenum along the afferent loop and reach to the orifice of papilla. Adjust the papilla and arciform knife for convenience of cannulation
All patients signed the research informed consent form, and then ERCP was performed under fluoroscopic control using a forward-viewing double-channel gastroscope (Pentax EG-3831T; channel diameter, $2.8 \mathrm{~mm}$ and $4.2 \mathrm{~mm}$ ). It was easy to distinguish the afferent loop and efferent loop of patients when a forward-viewing gastroscope was used. The afferent loop is always filled with bile and bubbles. Also, the afferent loop has a thin enteric cavity with abundant bile and bubbles attached to the intestinal walls, while for the efferent loop, the enteric cavity is larger and the intestinal wall is cleaner. When the gastroscope was placed through the afferent loop, the papilla was turned to face the gastroscope. The body position of the patients could be changed or repeated rotation, clasping, pulling, and pushing could be used to deliver gastroscope to the papilla if the advance of the gastroscope was hindered. The view of gastroscope and catheter were in line with the common bile duct when the selective bile duct cannulation was performed, but the location of papilla was indeterminate because it was determined by the direction of gastrointestinal anastomosis. At this moment, the gastroscope was adjusted to make sure that the papilla was in the upper-middle or lower-middle part of the field of view. Most of the catheters and arciform knives always have flexible tips, which make the success rate of cannulation into the pancreatic duct increase significantly. To avoid this problem, the body position of the patients could be changed during the cannulation, such as left lateral position, horizontal position, or prone position, and catheters with straight tips were preferable. The angle of the catheter could only be altered by adjusting the screw; due to no elevator in the double-channel gastroscope, pre-cut sphincterotomy could be performed when necessary. The incision for a standard papillotome is always locate at 9-3 o'clock positions, and the direction of the gastroscope access after Billroth II gastrectomy is opposite to that, which may lead to a hard sphincterotomy. In addition, we could rotate the papillotome for a proper angle (Figure 1). The double-channel gastroscope has two working channels $(4.2-\mathrm{mm}$ diameter, $2.8-\mathrm{mm}$ diameter). A catheter could be inserted into the $2.8-\mathrm{mm}$ diameter channel to support the papilla or change the direction of the orifice by pulling the papilla with biopsy forceps when there is difficulty in cannulation, with subsequent insertion of the catheter into the bile duct through the $4.2 \mathrm{~mm}$-diameter channel (Figures 2 and 3). Following selective cannulation, endoscopic sphincterotomy (EST), endoscopic papillary balloon dilatation (EPBD), endoscopic nose-bile drainage (ENBD), common bile duct stone removal, biliary drainage, and/or stent implantation in the bile duct were 
performed. The guidewire was retained in the bile duct if possible when removing or fragmenting the stones, to reduce the difficulty and time of re-cannulation. For the large stones (diameter $>1.0 \mathrm{~cm}$ ) mechanical lithotripsy was attempted, to fragment the stones before removing them. The Boston Scientific Stone Retrieval Basket could be delivered along the guidewire to make it easier for the access. If complete stone removal was not achieved in one session, biliary drainage was performed to relieve the obstruction and remove the stone at another proper time. For patients with a malignant mass at the distal end of the common bile duct, a metal stent or a plastic stent for the drainage was inserted into the bile duct after sphincterotomy (Figure 4).

\section{Results}

There were 3 cases of emergency treatments and 15 cases of elective operations. Out of 18 patients, endoscopic treatment was successful in 15 patients, resulting in a success rate of $83 \%$.

Endoscopic sphincterotomy was performed in 14 patients and EPBD was performed in 1 patient. Complete stone removal was achieved in 13 (86.7\%) patients and 9 patients' stones were removed in one session. Two patients were given stone removal for a second time after confirmation by ENBD. In 2 cases, large stones were discovered during the first session of radiography, but stone removal was not performed and only a nasobiliary drainage tube was retained due

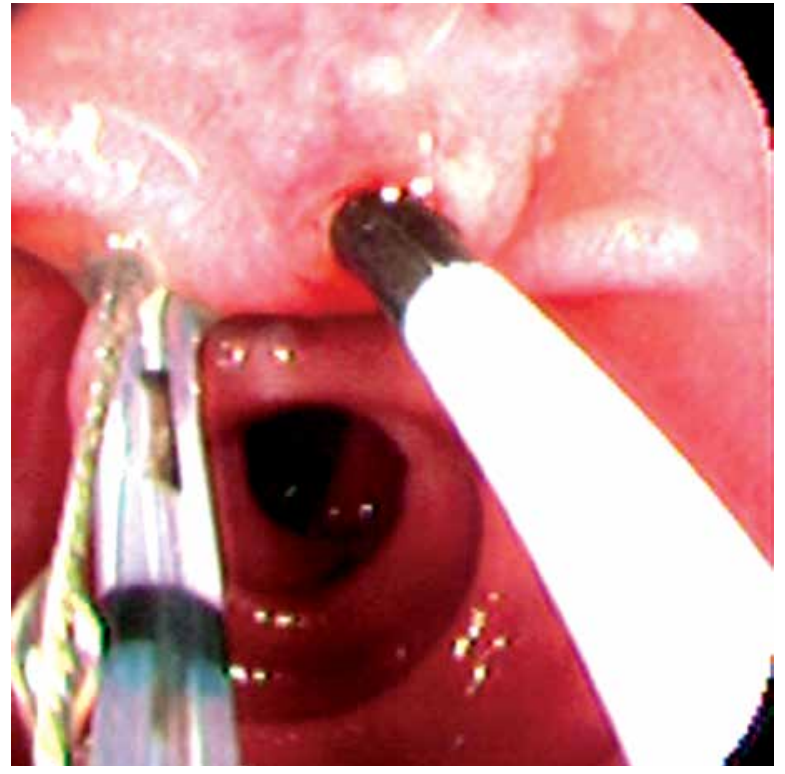

Figure 2. When there is difficulty in cannulation, a second channel can be used. Insert a catheter through the 2.8-mm channel to support the papilla, or change the direction of the orifice by pulling the papilla with biopsy forceps, then insert the catheter into the bile duct through the 4.2-mm channel

to severe biliary tract infection. Then the stones were removed for the second time after the conditions of the patients were resolved. In the 2 cases with failed

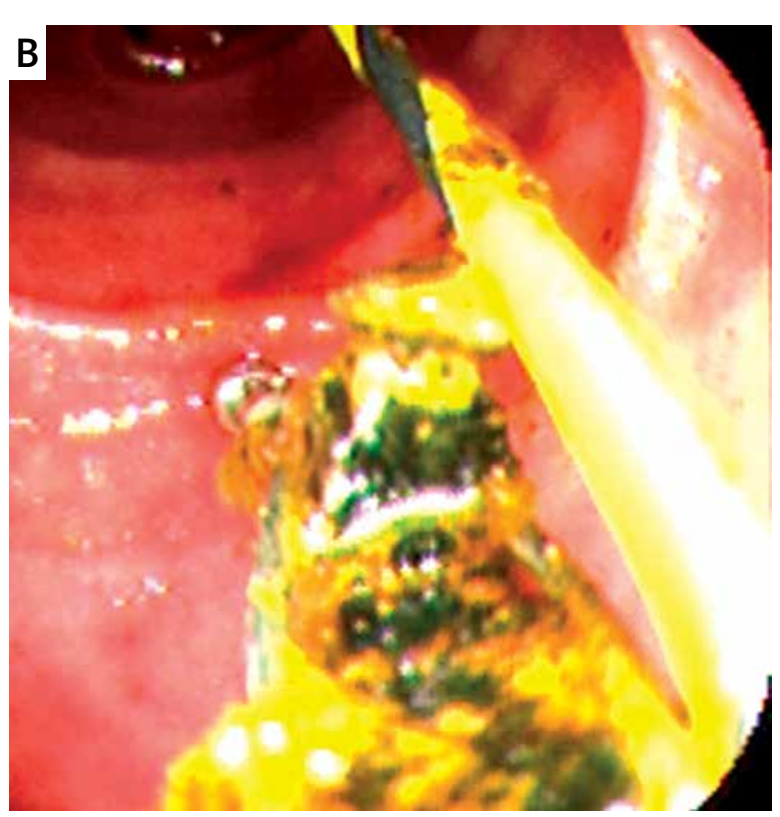

Figure 3. A - Filling defect of the common bile duct could be seen in the radiography, the guide wire is inserted through one channel and the Stone Retrieval Basket could be delivered through another channel. B - The guide wire is visible in one channel under endoscope, and the Stone Retrieval Basket for stone removal in another channel 

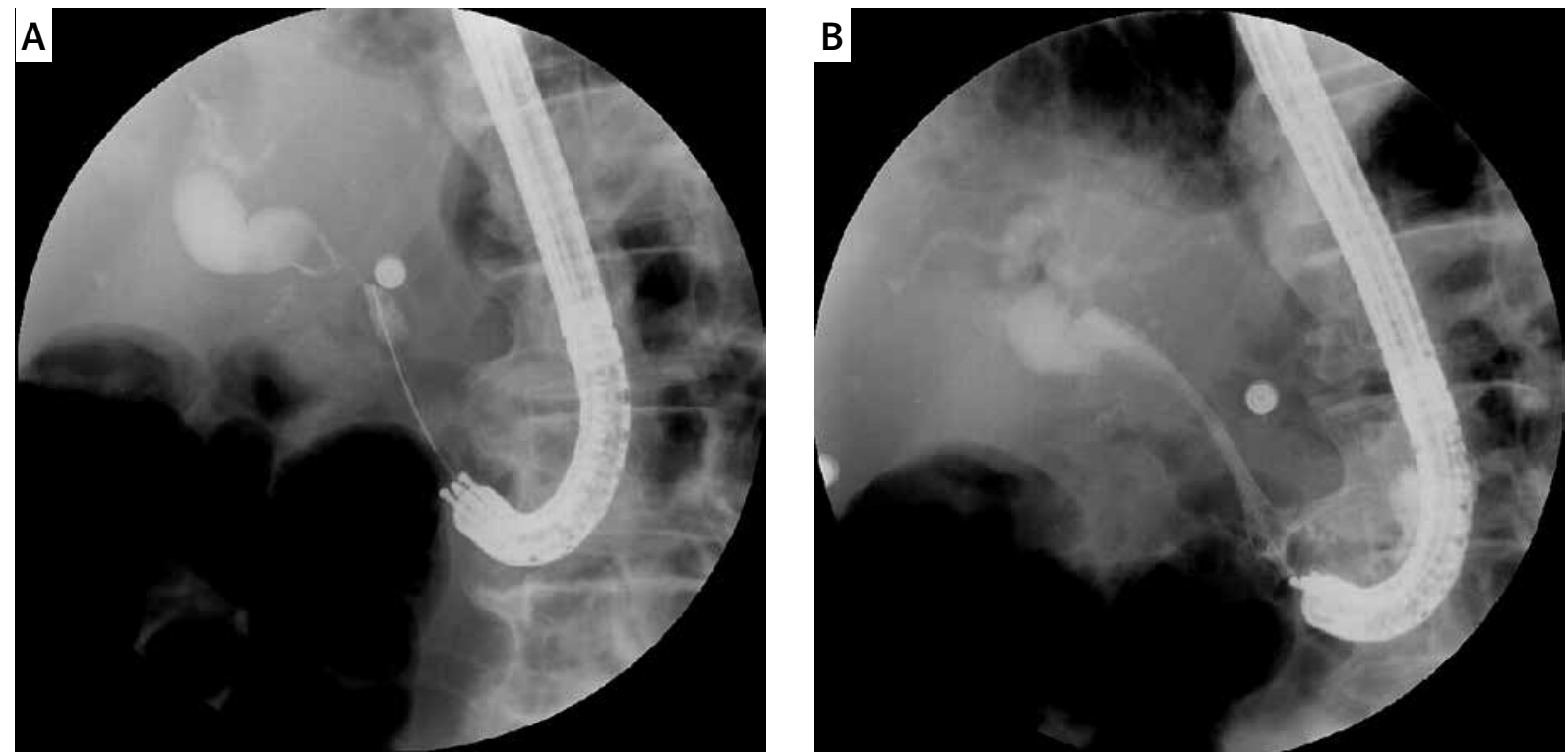

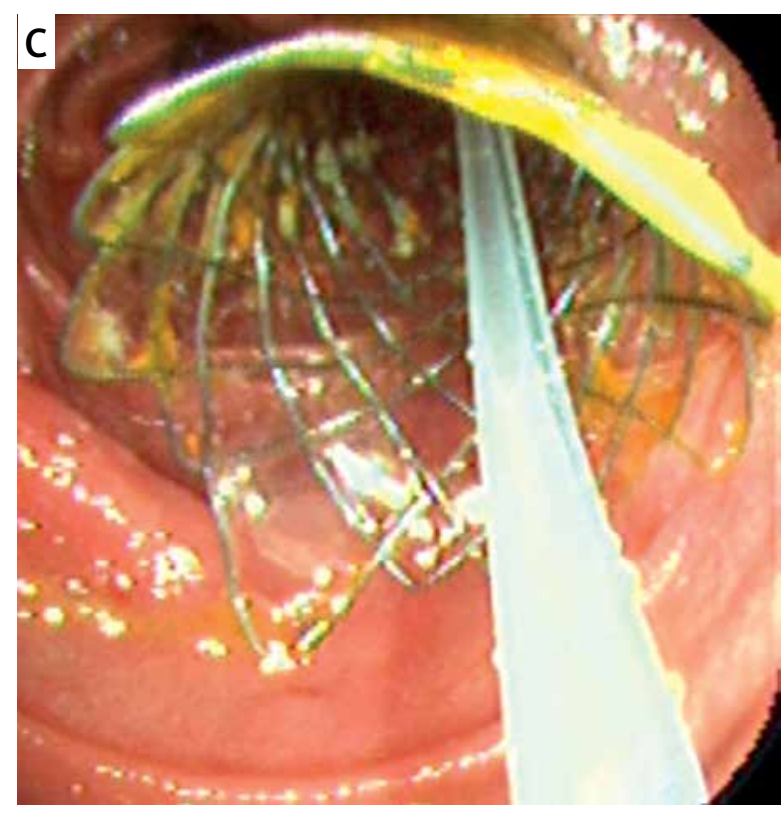

surgeries, major papilla was not visible in 1 case due to a long afferent loop, and the other 1 had malposition of major duodenal papilla-induced selective bile duct cannulation failure. There were three cases preoperatively diagnosed with a malignant mass at the distal end of the common bile duct and papillary carcinoma. Successful biliary stent insertion was performed in the two cases with a malignant mass at the distal end of the common bile duct, and for the other case of papillary carcinoma, ERCP was unsuccessful because of tumour infiltration. The preoperative diagnoses of the patients were all consistent with those after the operation. The clinical symptoms were significantly resolved after operation. Only 2 patients had abdominal pain and other
Figure 4. A - Proximal end dilation of the common bile duct could be seen in the radiography, there is no development of terminal bile duct. B - The metal stent is implanted through the double-channel gastroscope, and the middle segment is transformed by pressure, but the upper and lower end opening is good, the contrast agent could be delivered through the stent to the intestinal tract. $\mathbf{C}$ - The form of the stent at the end of the intestinal tract is good under the endoscope, but there is bile and contrast agent outflow

symptoms as well as elevated serum amylase by three times after endoscopic treatment. All the symptoms were resolved after conservative medical therapy, with the longest duration of 1 week. One patient had minor bleeding after the ERCP, and fresh blood was drained out by ENBD, which was successfully managed with conservative treatment. There was no perforation or death related to the procedures in any patient. Eleven cases with bile duct stones were followed up after treatment for 3-24 months, 5 of which had recurrent common bile duct stones and were successfully removed under endoscopy again. There were 3 cases of death occurring 3-12 months after the operation because of tumours (Table II). 
Table II. Biliary diseases treated with double-channel gastroscope after Billroth II gastrectomy

\begin{tabular}{|c|c|c|c|c|c|c|c|c|c|}
\hline No. & Diagnoses & $\begin{array}{l}\text { Succeed } \\
\text { cases }\end{array}$ & $\begin{array}{l}\text { Failed } \\
\text { cases }\end{array}$ & $\begin{array}{l}\text { Treatment } \\
\text { method }\end{array}$ & $\begin{array}{l}\text { Times of } \\
\text { treatment }\end{array}$ & $\begin{array}{l}\text { Recurrent } \\
\text { stone }\end{array}$ & Bleeding & Pancreatitis & $\begin{array}{l}\text { Digestive } \\
\text { tract } \\
\text { perforation }\end{array}$ \\
\hline 1 & Choledocholithiasis & $\sqrt{ }$ & & EST & 1 & & & & \\
\hline 2 & Choledocholithiasis + gallstone & $\sqrt{ }$ & & EST & 1 & $\sqrt{ }$ & & & \\
\hline 3 & Choledocholithiasis + gallstone & $\sqrt{ }$ & & ENBD & 2 & & & & \\
\hline 4 & Choledocholithiasis & & $\sqrt{ }$ & & & & & & \\
\hline 5 & Choledocholithiasis + gallstone & $\sqrt{ }$ & & EST & 1 & & & & \\
\hline 6 & Choledocholithiasis & $\sqrt{ }$ & & EST & 2 & $\sqrt{ }$ & & & \\
\hline 7 & Choledocholithiasis + gallstone & $\sqrt{ }$ & & EST & 1 & & & $\sqrt{ }$ & \\
\hline 8 & Choledocholithiasis + gallstone & & $\sqrt{ }$ & & & & & & \\
\hline 9 & Bile duct carcinoma & $\sqrt{ }$ & & Metal stent & 1 & & & & \\
\hline 10 & Choledocholithiasis + gallstone & $\sqrt{ }$ & & EST & 1 & $\sqrt{ }$ & & & \\
\hline 11 & Choledocholithiasis & $\sqrt{ }$ & & EBD & 1 & & $\sqrt{ }$ & & \\
\hline 12 & Choledocholithiasis & $\sqrt{ }$ & & ENBD & 2 & $\sqrt{ }$ & & & \\
\hline 13 & Choledocholithiasis + gallstone & $\sqrt{ }$ & & EST & 2 & & & & \\
\hline 14 & Bile duct carcinoma & $\sqrt{ }$ & & Metal stent & 1 & & & & \\
\hline 15 & Duodenal papilla carcinoma & & $\sqrt{ }$ & & & & & & \\
\hline 16 & Choledocholithiasis + gallstone & $\sqrt{ }$ & & EST & 1 & & & $\sqrt{ }$ & \\
\hline 17 & Choledocholithiasis + gallstone & $\sqrt{ }$ & & EST & 1 & & & & \\
\hline 18 & Choledocholithiasis & $\sqrt{ }$ & & EST & 1 & $\sqrt{ }$ & & & \\
\hline Total & 18 & 15 & 3 & & 19 & 5 & 1 & 2 & 0 \\
\hline
\end{tabular}

EST - endoscopic sphincterotomy, ENBD - endoscopic nose-bile drainage.

\section{Discussion}

The incidence rate of bile duct infection and bile duct stone after Billroth II gastrectomy was 16-42\% [1], mostly occurring at a senior age - average 65 years $[2,3]$, as reported in the foreign literature. Patients with Billroth II gastrectomy have a higher incidence of biliary diseases, mainly due to the following: 1) injuries of the pneumogastric nerve at the lesser curvature during the gastroenterostomy - in particular the block of hepatic plexus, celiac branches, and pyloric plexus - lead to impairment of gallbladder contractibility, and the cholestasis induced by sphincter of Oddi dysfunction, bile duct infection, and the change of bile composition also contribute to the formation of bile duct stones. Kwon et al. [4] reported that the incidence rate of gallbladder diseases was $22.8 \%$ after vagotomy, while the figure was $2 \%$ if selective vagotomy is adopted. 2 ) The lithogenesis might be related to the bile drainage problems induced by adhesion between the gallbladder, extrahepatic bile duct, duodenal stump, and adjacent organs. 3) The reduction of gastric acid secretion induced by gastrectomy may lead to a decrease in the bacteria in the gastrointestinal tract, and the retrograde motion of bacteria in the duodenum to the bile duct may induce bile duct infection, thus increasing the incidence rate of the primary choledocholithiasis. 4) The short or long afferent loop may induce retention of a large amount of food, which leads to bile duct infection and choledocholithiasis [5].

Most of the patients were at a senior age, and there was celiac adhesion, which meant that the operations were high risk and difficult. It was suggested that the endoscopic treatment be adopted in the first place. Laparotomy could be used if the primary treatment failed. ERCP is one of the best methods to diagnose bile duct diseases. However, it is quite risky to deliver the endoscope to the duodenal papilla because of the altered anatomy after operation. Consequently the author did not recommend ERCP for diagnosis, but for treatment. The foreign literature indicated that the success rate of endoscopic treatment was 55-88\% [6]. A side-viewing endoscope or a forward-viewing endoscope could be used. In this study, all of the ERCPs were performed using a forward-viewing double-channel gastroscope 
with good results (83\%). The main reasons are as follows: 1) a double-channel gastroscope is easier to deliver, while the afferent loop and efferent loop could be distinguished easily. 2) A double-channel gastroscope is easier to rotate, clasp, pull, and push to reach the papilla through the afferent loop. 3) The view of the forward-viewing gastroscope and biliary tract were almost in the same direction, so it is easier to adjust the catheter during cannulation. 4) When the papilla is at an undesired location, the gastroscope can be adjusted to make sure the duodenal papilla is in the upper-middle or lower-middle part of the field of view for the convenience of cannulation. 5) When there is difficulty in cannulation, another channel can be used to insert a catheter or biopsy forceps to hold the papilla, to facilitate selective cannulation. The larger channel of the double-channel gastroscope is $4.2 \mathrm{~mm}$ in diameter, which could deliver the metal stent conveyer and mechanical apparatus for the stones, thus increasing the feasibility of endoscopic treatment and the selectivity of relevant mechanical devices [7]. For cases in which the gastroscope cannot reach the orifice of papilla due to a long afferent loop, the foreign literature reported that a double-balloon enteroscope was used for ERCP in patients with Billroth II gastrectomy, which is recommended $[8,9]$. Cannulation failure is mainly induced by the lack of a special knife for the forward-viewing gastroscope [10, 11].

We made a pre-cut to the papillary muscle in four failed bile duct intubation cases, the direction of the pre-cut is the mouth side of the orifice in ampulla. The view is from the distal to the proximal direction of the duodenum, we cut from the duodenum papilla to the proximal bile duct. Mechanical lithotripsy was attempted in order to fragment the stones before removing them before August 2010 in our hospital, followed by four large stones that were treated by plus expansion. No serious complications occurred, but as there are few cases, its security needs further validation.

It is reported in the foreign literature that the incidence rate of complications during ERCP after Billroth II gastrectomy was $8 \%$ and the death rate was $2 \%$ [12]. In our hospital, the incidence rate of complications during ERCP was $16.7 \%$ (3/18) - mainly pancreatitis and delayed bleeding - which were not consistent with the reports overseas (intestinal perforation) [13-15]. We think that this difference was induced by the application of a forward-viewing gastroscope, which could significantly reduce the incidence rate of the intestinal perforation. It is believed that the incidence rate of bleeding and perforation of EPBD is lower than that of EST [16]. For large stones (>10 mm) and multiple stones (>3), the removal is difficult, and EPBD is not applicable in senior patients. Consequently, in this report only 1 out of 15 patients was given EPBD.

\section{Conclusions}

The application of a double-channel gastroscope could reduce the operative difficulty of ERCP in patients with Billroth II gastrectomy, and a higher success rate could be achieved. Meanwhile, severe complications could be prevented. The double-channel gastroscope can be safely and effectively used to perform ERCP in patients with Billroth II gastrectomy. The long afferent loop induced by Billroth II gastrectomy and the limitation of a cannulation device are still the main causes for the failure of the operation, while the double-channel gastroscope used for ERCP still has similar complications as normal ERCP.

\section{Conflict of interest}

The authors declare no conflict of interest.

\section{References}

1. Fan JG, Zhu J, Li XJ, et al. Prevalence of and risk factors for fatty liver in a general population of Shanghai, China. J Hepatol 2005; 43: 508-14.

2. Bergman J, van Berkel AM, Bruno MI, et al. A randomized trial of endoscopic balloon dilation and endoscopic sphincterotomy for removal of bile duct stones in patients with a prior Billroth II gastrectomy. Gastrointest Endosc 2001; 53: 19-26.

3. Kianicka B, Díte P, Piskac P. Endoscopic diagnostics and management of pancreatic-biliary disorders in patients after Billroth II gastric resection. Vnitr Lek 2009; 55: 1043-50.

4. Kwon AH, Inui H, Imamura A, et al. Laparoscopic cholecystectomy and choledocholithotomy in patients with a previous gastrectomy. J Am Coll Surg 2001; 193: 614-9.

5. Desbeaux A, Hec F, Andrieux S, et al. Risk of biliary complications in bariatric surgery. J Visc Surg 2010; 147: e217-20.

6. Sümegi J. Endoscopic retrograde cholangio-pancreatography after conventional Billroth II resection. Orv Hetil 2004; 145 : 2425-30.

7. Spaun GO, Zheng B, Martinec DV, et al. Bimanual coordination in natural orifice transluminal endoscopic surgery: comparing the conventional dual-channel endoscope, the R-Scope, and a novel direct-drive system. Gastrointest Endosc 2009; 69: 39-45.

8. Albrecht H, Konturek PC, Diebel H, et al. Successful interventional treatment of postoperative bile duct leakage after Billroth II resection by unusual procedure using double balloon enteroscopy. Med Sci Monit 2011; 17: CS29-33.

9. Itoi T, Ishii K, Sofuni A, et al. Single-balloon enteroscopy-assisted ERCP in patients with Billroth II gastrectomy or Roux-en-Y anastomosis (with video). Am J Gastroenterol 2010; 105: 93-9.

10. van Buuren HR, Boender J, Nix GA, van Blankenstein M. Needle-knife sphincterotomy guided by a biliary endoprosthesis in Billroth II gastrectomy patients. Endoscopy 1995; 27: 229-32. 
11. Al-Kawas FH, Geller AJ. A new approach to sphincterotomy in patients with Billroth II gastrectomy. Gastrointest Endosc 1996; 43: 253-5.

12. Faylona JM, Qadir A, Chan AC, et al. Small-bowel perforations related to endoscopic retrograde cholangiopancreatography (ERCP) in patients with Billroth II gastrectomy. Endoscopy 1999; 31: 546-9.

13. Mosca S. How can we reduce complication rates and enhance success rates in Billroth II patients during endoscopic retrograde cholangiopancreatography? Endoscopy 2000; 32: 589-90.

14. Hintze RE, Adler A, Veltzke W, Abou-Rebyeh H. Endoscopic access to the papilla of Vater for endoscopic retrograde cholangiopancreatography in patients with Billroth II or Roux-en-Y gastrojejunostomy. Endoscopy 1997; 29: 69-73.

15. Avgerinos DV, Llaguna OH, Lo AY, et al. Management of endoscopic retrograde cholangiopancreatography: related duodenal perforations. Surg Endosc 2009; 23: 833-8.

16. Cairns SR. Endoscopic balloon sphincteroplasty: use in a patient with a Billroth II gastrectomy. Endoscopy 1996; 28: 790.

Received: 6.12.2014

Accepted: 10.04 .2015 